\title{
Percepção de competência, em situação de atividades diversificadas na perspectiva dos professores e de seus alunos com e sem deficiência física
}

\author{
Perception of competence in the situation of diversified activities in the \\ perspective of the teachers and of their students with and without physical \\ deficiency
}

\author{
Mirela Moreno Almeida de Andrade ${ }^{1}$, Camila Boarini dos Santos ${ }^{2}$, \\ Rita de Cássia Tibério Araújo ${ }^{3}$
}

doi: http://dx.doi.org/10.11606/issn.2238-6149.v29i3p246-253

Andrade M.M.A, Santos CB, Araújo RCT. Percepção de competência, em situação de atividades diversificadas na perspectiva dos professores e de seus alunos com e sem deficiência física. Rev Ter Ocup Univ São Paulo. 2018 set.-dez.;29(3):246-53.

RESUMO: Muito se debate sobre a necessidade de um ambiente diferenciado, que disponibilize recursos adaptados às necessidades da pessoa com deficiência, no entanto, acredita-se que deva ser dada atenção à relação indivíduo-tarefa-ambiente, uma vez que a expressão de competências emerge da sua interação com o meio. Este estudo teve o objetivo de identificar características da percepção de competência, na perspectiva dos professores e alunos com e sem deficiência física. Participaram deste estudo dezoito alunos e nove professores. Os dados foram coletados com o Perceived Eficacy and Goal Setting System e a análise foi realizada pelo teste de Mann-Whitney. Os resultados demonstraram que a percepção dos professores para a competência dos alunos sem deficiência foi mais favorável do que a percepção para os alunos com deficiência. No entanto, os valores de dispersão, demonstraram que em todas as análises, os alunos sem deficiência apresentaram uma autopercepção mais favorável. Conclui-se que há diferença na percepção de competência tanto na perspectiva dos professores quanto dos próprios alunos, sendo esta desfavorável em virtude da presença de deficiência física.

Descritores: Educação especial; Percepção; Competência.
Andrade M.M.A, Santos CB, Araújo RCT. Perception of competence in the situation of diversified activities in the perspective of the teachers and of their students with and without physical deficiency. Rev Ter Ocup Univ São Paulo. 2018 SeptDec;29(3):246-53.

ABSTRACT: There is much debate about the need for a differentiated environment, which provides resources adapted to the needs of the disabled, however, attention must be paid to the individual-task-environment relationship, since the expression of competences emerges from its interaction with the middle. This study aimed to identify characteristics of the perception of competence, from the perspective of teachers and students with and without physical disabilities. Participated in this study were eighteen students and nine teachers. The data were collected with the Perceived Efficiency and Goal Setting System and the analysis performed by the Mann-Whitney test. The results showed that the teachers' perception of the competence of students without disabilities was more favorable than the perception for students with disabilities. However, the dispersion values showed that in all analyzes, students without disabilities had a more favorable self-perception. It is concluded that there is a difference in the perception of competence both from the teachers perspective and from the students themselves, which is unfavorable due to the presence of physical disability.

Keywords: Education, special; Perception; Competence.

Artigo é parte integrante da dissertação "Percepção de competência na execução de atividades realizadas por escolares com e sem deficiência".

1. Terapeuta Ocupacional. Doutoranda do Programa de Pós-Graduação em Educação pela Universidade Estadual Paulista Júlio de Mesquita Filho - UNESP, São Paulo, SP, Brasil. ORCID: https://orcid.org/0000-0002-4588-4132. Email:midejulia@gmail.com.

2. Terapeuta Ocupacional. Mestranda em Educação e bolsista CAPES pela Universidade Estadual Paulista Júlio de Mesquita Filho UNESP, São Paulo, SP, Brasil. ORCID: https://orcid.org/0000-0001-5594-0305. Email: camilaboarini@hotmail.com.

3. Terapeuta Ocupacional. Professora Doutora do Curso de Terapia Ocupacional - Departamento de Fisioterapia e Terapia Ocupacional da Universidade Estadual Paulista Júlio de Mesquita Filho - UNESP, São Paulo, SP, Brasil. ORCID: https://orcid.org/0000-0002-14631175. Email: ritac@marilia.unesp.br.

Endereço para correspondência: Rua: Wady Butara nº 1112. Jardim São Domingos, Marília, SP. CEP: 17514730. 


\section{INTRODUÇÃO}

análise da percepção de competência é um objeto de estudo de fundamental importância, especialmente quando o público alvo da educação especial está contemplado.

O termo competência pode ser descrito como o desempenho ou execução habilidosa de ações propositais relacionadas com as demandas do ambiente, implicando resultados aceitáveis, porém não necessariamente perfeitos. Quando o indivíduo torna-se capaz de realizar adequadamente diferentes tarefas e de se relacionar de maneira eficiente com o meio, a ponto de ser possível o aprimoramento de habilidades de acordo com o feedback recebido em resposta às suas ações, pode-se dizer que tratase de alguém competente ${ }^{1}$.

Nessa mesma perspectiva, a competência pode ser compreendida como a interação efetiva com os meios físico social, à medida que ser competente significa possuir habilidades suficientes e ou adequadas para responder às demandas da situação ou tarefa ${ }^{2}$.

Para Trombly e Radomski ${ }^{3}$ o senso de competência permite que as pessoas acreditem na sua capacidade de controle sobre o meio físico e social ao invés de serem por eles controladas. A competência na realização de atividade está relacionada ao resultado da ação, que, por sua vez, depende de como é percebido pela própria pessoa e pela audiência, tendo como base os padrões de desempenho, nos limites considerados aceitáveis para caracterizar a competência.

A competência de um indivíduo, para que a sua participação seja efetiva no ambiente, como, por exemplo, no ambiente escolar, deve ultrapassar a execução de atividades específicas e atingir o nível de consistência e generalização a outras situações tanto com relação a outras atividades quanto com relação a outros contextos.

Os indivíduos modificam os seus comportamentos e atitudes em face das tarefas e do modo como percebem a sua competência pessoal e de acordo com a expectativa de sucesso. Os alunos que desvalorizam a sua competência, fragilizam a confiança nas suas capacidades ${ }^{4-7}$. A fragilidade pode ser apenas subjetiva ou, em longo prazo, tornar-se objetiva, à medida que a ideia ilusória de incompetência pode levar a subestimação das reais competências, no entanto, caso haja a valorização excessiva da competência isso resultará, igualmente, em investimento deslocado do foco de atenção porque em vez de gerir o processo de aprendizagem, passa a centrar-se no resultado $0^{8,9}$.

Um aluno pode estabelecer um nível de competência para determinada atividade em um momento circunstanciado e outro aluno pode estabelecer outro nível de competência para a mesma atividade, assim como o seu professor pode ter a expectativa de competência com relação a ambos, muito diferente daquelas estabelecidas por eles.

Segundo Ferreira ${ }^{10}$, a autopercepção de competência no ambiente escolar estabelece relação com a experiência de realizar e completar uma atividade de forma eficaz e que gere aprendizagem, considerando que a necessidade de exercer o controle de suas ações, leva o indivíduo à necessidade de buscar autonomia.

O professor exerce um dos papeis mais importantes da educação inclusiva, pois a partir da sua participação efetiva influenciada por muitas variáveis, destacando-se dentre elas a compreensão das características e necessidades especiais dos alunos com deficiência, o treinamento para a utilização de estratégias de ensino inclusivas e o desenvolvimento de atitudes sociais intrinsecamente favoráveis à inclusão $0^{11-13}$.

As atitudes sociais dos professores são determinantes para a promoção do ensino inclusivo, entretanto, geralmente os professores não recebem formação especializada para lidar com a situação de ter, em sua sala de aula, um aluno com deficiência. E apenas a capacitação com relação à compreensão das características e necessidades deste aluno e a utilização de ampla variedade de recursos não seriam suficientes, mas, sim, teria que ser construída uma nova visão de ensino e de aprendizagem, fundada em atitudes favoráveis à inclusão ${ }^{14}$.

A inclusão dos alunos com deficiência, na escola comum do ensino regular, implica concepções e atitudes positivas dos educadores para que se alcance um processo de aprendizagem e desenvolvimento realmente produtivo para todos os alunos.

As atitudes sociais dos professores são apontadas como uma das variáveis mais críticas para o sucesso da inclusão e se tornam ainda mais importantes ao considerarmos que a resposta que colegas de classe emitem diante de aluno com deficiência pode ser parcialmente determinada pela postura assumida pelo professor ${ }^{15,16}$.

Assim sendo, podemos considerar que além de atitudes sociais positivas frente à inclusão, o professor precisa conhecer, de fato, os seus alunos e isto se torna ainda mais importante quando nos referimos aos alunos com deficiência, pois, as suas necessidades e capacidades podem ser camufladas diante da coletividade da sala de aula e, desta forma, as estratégias e recursos utilizados pelo professor no processo de ensino e aprendizagem podem ser insuficientes para contemplar as diferenças que a heterogeneidade da sala de aula impõe.

No que se refere às oportunidades de experiência 
escolar, entende-se que a educação, por princípio, deve favorecer as oportunidades de sucesso daqueles sobre os quais ela se responsabiliza, e, nessa perspectiva, a inclusão de alunos com deficiência na rede comum do ensino é um grande avanço, uma vez que o desenvolvimento psíquico depende das interações mediadas pelo outro, cabendo à escola um papel significativo no desenvolvimento do aluno, de forma a conduzi-lo para estágios mais complexos de interação, comportamento e funcionamento intelectual.

Ao considerarmos o ambiente da educação inclusiva, identificamos como fator fundamental a presença de um professor atento e disposto a auxiliar os alunos com deficiência a adquirirem competência na realização das atividades propostas na escola. Atribuímos a este professor mediador a responsabilidade de avaliar as capacidades e necessidades dos seus alunos, e, para que isto possa ocorrer, acreditamos que seja fundamental conhecer qual a percepção do professor a respeito da competência dos seus alunos na realização de atividades diversificadas bem como conhecer a autopercepção de competência tanto dos alunos com deficiência quanto dos alunos sem deficiência, que convivem no mesmo espaço, nas mesmas atividades desempenhadas no cotidiano escolar.

Nessa perspectiva, o objetivo geral deste estudo foi identificar as características da percepção de competência, em situação de atividades diversificadas na perspectiva dos professores e de seus alunos com e sem deficiência física, tendo como objetivos específicos, identificar se existem diferenças nas percepções de competência mediante análises comparativas da percepção dos professores com relação aos alunos com e sem deficiência, da autopercepção dos alunos com e sem deficiência e na comparação entre a percepção dos professores e a autopercepção dos alunos.

\section{PROCEDIMENTOS METODOLÓGICOS}

O estudo foi aprovado pelo Comitê de Ética em Pesquisa da Faculdade de Filosofia e Ciências da UNESP - Campus de Marília/SP seguindo as recomendações vigentes na resolução CNS 196/96, conforme protocolo ${ }^{\circ}$ 1119/2014. É parte de uma pesquisa maior, realizada para dissertação de mestrado do Programa de Pós-Graduação em Educação da Faculdade de Filosofia e Ciências da UNESP - Campus de Marília/SP e apresenta desenho de estudo descritivo conforme classificação de Triviños ${ }^{17}$.

Participaram desta pesquisa dezoito alunos do ciclo I do ensino fundamental matriculados em classes do primeiro ao quarto ano, dos quais nove com deficiência física e nove sem deficiência, com idade variando de seis a doze anos. Os diagnósticos foram diversos, porém na maioria dos casos tratava-se de alunos com Paralisia Cerebral (sete casos), sendo os demais diagnósticos a Mielomeningocele e Mal Formação Congênita. Além dos alunos, participaram da pesquisa nove professoras dos respectivos alunos, sendo cada um delas responsável por um aluno com e sem deficiência da mesma sala de aula.

Os critérios de inclusão abrangeram os alunos com deficiência física, identificados pelo cadastro disponível na Secretaria de Educação matriculados no ensino fundamental da rede regular, excluindo deste estudo os alunos com distúrbios auditivos, visuais e/ou intelectuais associados.

A pesquisa foi realizada em seis escolas de ensino fundamental da rede regular de ensino, localizadas em um município do interior do Estado de São Paulo.

Como instrumento de coleta de dados utilizou-se o Perceived Eficacyand Goal Setting System (PEGS) que foi traduzido e adaptado para a cultura Brasileira por Ruggio (2008) em trabalho de dissertação apresentada ao Programa de Mestrado em Ciências da Reabilitação da Escola de Educação Física, Fisioterapia e Terapia Ocupacional da Universidade Federal de Minas Gerais, para a obtenção do título de Mestre em Ciências da Reabilitação. O objetivo do instrumento é possibilitar que crianças com ou sem alguma deficiência relatem sua autopercepção em relação à competência na realização das atividades do dia $\mathrm{a} \mathrm{dia}^{18}$. O PEGS pode ser utilizado com diferentes diagnósticos, sendo mais apropriado para crianças entre seis e nove anos de idade cronológica ou nível de desenvolvimento compatível para esta faixa etária ${ }^{19,20}$. É composto por três partes: o protocolo de entrevista da criança, um questionário de pais e outro de professores. Diante da natureza deste estudo, o questionário para pais não foi utilizado ${ }^{18}$.

Inicialmente, foi encaminhado à Secretaria de Educação um pedido de autorização e de identificação dos alunos com deficiência física matriculados no ciclo I do Ensino Fundamental e suas respectivas escolas. Foram apresentados vinte e cinco alunos com deficiência física, dos quais, nove foram selecionados a partir do aceite da escola e dos pais para participarem da pesquisa conforme apresentado na Figura 1.

A aplicação do protocolo com os alunos ocorreu individualmente e seguiu a orientação apresentada pelo autor. O tempo médio de aplicação com os alunos foi de vinte minutos e não houve diferença no tempo de duração do procedimento entre o grupo com deficiência e o sem deficiência. Nos casos de alunos que utilizavam cadeira de rodas, foram utilizados os cartões específicos para esta condição. 
A análise estatística com o cálculo dos quartis para as medidas de dispersão se fez necessária nos grupos onde não houve diferença estatisticamente significante, pois desta forma foi possível identificar as diferenças entre eles com relação aos valores de escore calculados conforme demonstrado na Tabela 2. Acredita-se que em outros estudos similares, porém com um número maior de participantes, talvez não seja necessária a análise por meio dos cálculos dos quartis e apenas o teste estatístico não paramétrico seja suficiente.

Tabela 2- Medidas de dispersão com os Quartis da análise obtida no PEGS

\begin{tabular}{|c|c|c|c|c|c|}
\hline & & Deficiência & Min.-Max. & Md & Q1-Q3 \\
\hline \multirow{2}{*}{ Geral } & \multirow{2}{*}{ Professor } & Com & $39-60$ & 45 & $42-54$ \\
\hline & & Sem & $56-60$ & 59 & $58-60$ \\
\hline \multirow{2}{*}{ Brincar } & \multirow{2}{*}{ Professor } & Com & $13-24$ & 19 & $16-22$ \\
\hline & & Sem & $23-24$ & 24 & $24-24$ \\
\hline \multirow{2}{*}{ Trabalho escolar } & \multirow{2}{*}{ Professor } & Com & $15-26$ & 22 & $21-23$ \\
\hline & & Sem & $23-27$ & 26 & $26-27$ \\
\hline \multirow{2}{*}{ Atividades de autocuidado } & \multirow{2}{*}{ Professor } & Com & $3-9$ & 8 & $7-8$ \\
\hline & & Sem & $9-9$ & 9 & $9-9$ \\
\hline \multirow{2}{*}{ Geral } & \multirow{2}{*}{ Alunos } & Com & $52-62$ & 56,5 & $55-59,5$ \\
\hline & & Sem & $56-60$ & 58 & $58-60$ \\
\hline \multirow{2}{*}{ Brincar } & \multirow{2}{*}{ Alunos } & Com & $13-24$ & 22,5 & $22-24$ \\
\hline & & Sem & $24-24$ & 24 & $24-24$ \\
\hline \multirow{2}{*}{ Trabalho escolar } & \multirow{2}{*}{ Alunos } & Com & $19-27$ & 26 & $25-26$ \\
\hline & & Sem & $25-27$ & 26 & $25-27$ \\
\hline \multirow{2}{*}{ Atividades de autocuidado } & \multirow{2}{*}{ Alunos } & Com & $6-10$ & 8 & $8-9$ \\
\hline & & Sem & $9-9$ & 9 & $9-9$ \\
\hline \multirow{2}{*}{ Geral } & Professor & Com & $39-60$ & 45 & $42-54$ \\
\hline & Aluno & Com & $41-62$ & 55 & $55-59$ \\
\hline \multirow{2}{*}{ Brincar } & Professor & Com & $13-24$ & 22,5 & $22-24$ \\
\hline & Aluno & Com & $13-24$ & 22 & $19-24$ \\
\hline \multirow{2}{*}{ Trabalho escolar } & Professor & Com & $15-26$ & 22 & $21-23$ \\
\hline & Aluno & Com & $25-27$ & 26 & $25-26$ \\
\hline \multirow{2}{*}{ Atividades de autocuidado } & Professor & Com & $3-9$ & 7,5 & $7-8$ \\
\hline & Aluno & Com & $7-10$ & 8 & $8-9$ \\
\hline \multirow{2}{*}{ Geral } & Professor & Sem & $56-60$ & 59 & $58-60$ \\
\hline & Aluno & Sem & $56-60$ & 58 & $56-60$ \\
\hline \multirow{2}{*}{ Brincar } & Professor & Sem & $23-24$ & 24 & $24-24$ \\
\hline & Aluno & Sem & $24-24$ & 24 & $24-24$ \\
\hline \multirow{2}{*}{ Trabalho escolar } & Professor & Sem & $23-27$ & 26 & $26-27$ \\
\hline & Aluno & Sem & $25-27$ & 26 & $25-27$ \\
\hline \multirow{2}{*}{ Atividades de autocuidado } & Professor & Sem & $9-9$ & 9 & $9-9$ \\
\hline & Aluno & Sem & $9-9$ & 9 & $9-9$ \\
\hline
\end{tabular}




\section{RESULTADOS E DISCUSSÕES}

A Percepção dos professores em relação à competência de alunos com e sem deficiência, foi obtida a partir da análise de todas as avaliações dos professores que foram divididas em dois grupos, sendo um grupo de alunos com deficiência e o outro grupo de alunos sem deficiência.

O resultado obtido $(p=0.0040)$ demonstrou que houve diferença estatisticamente significante na avaliação entre os dois grupos e apontou para a avaliação mais favorável dos professores com relação à competência de seus alunos sem deficiência física nas atividades pesquisadas.

Diante dos resultados encontrados e do referencial teórico que nos remete à reflexão acerca da importância da figura do professor como mediador na relação aluno atividade - ambiente, devemos ponderar sobre a influência que estas percepções desfavoráveis com relação ao grupo de alunos com deficiência podem exercer na atuação do professor com estes alunos.

Acreditamos que a partir do conhecimento destas percepções o professor possa buscar novas soluções e estratégias visando o desenvolvimento pleno dos seus alunos com deficiência considerando o máximo das suas potencialidades. Esses resultados indicam que a prescrição e aplicação da Tecnologia Assistiva no contexto inclusivo seria importante para favorecer a funcionalidade do aluno com deficiência.

Estudo realizado por Pelosi e Nunes ${ }^{21}$ demonstrou que a implantação dos recursos de tecnologia assistiva na escola, com o objetivo de favorecer a inclusão de alunos com deficiência física, quando realizada de forma colaborativa entre profissionais da saúde e educação pode alcançar resultados muito positivos.

Em qualquer ambiente de aprendizagem, as respostas dos professores relativas ao sucesso ou fracasso da criança na realização de uma atividade têm impacto nas percepções de competência da mesma. O feedback advindo de professores ou amigos serve como fonte de referência sobre a adequação do desempenho da criança com deficiência, tendo efeito positivo ou negativo no autoconceito dependendo da importância e valor dado pela criança a esta experiência e a audiência ${ }^{22}$.

A análise comparativa da percepção dos dois grupos de alunos ocorreu considerando o escore total de cada grupo com base nas suas indicações de atividades realizadas com dificuldade e de atividades realizadas com facilidade, conforme procedimentos do protocolo aplicado. Seguindo o mesmo procedimento de análise, o tratamento estatístico dos dados foi realizado com o uso do teste Mann-Whitney com o objetivo de identificarmos se havia diferença na autopercepção de competência dos alunos com e sem deficiência ao realizarem as atividades investigadas. O resultado obtido $(\mathrm{p}=0.2224)$ demonstrou que não houve diferença estatisticamente significante entre os grupos pesquisados. No entanto, ao realizarmos a análise por meio do cálculo dos quartis com as medidas de dispersão, verificamos que os valores Q1 e Q3 bem como a mediana e o valor mínimo do grupo de alunos com deficiência são inferiores aos valores obtidos no grupo de alunos sem deficiência, e, desta forma, podemos inferir que a autopercepção dos alunos sem deficiência física é mais favorável com relação à sua competência quando comparada ao grupo de alunos com deficiência física. Ao identificarmos que os alunos com deficiência apresentam autopercepção de competência desfavorável quando comparada com a autopercepção dos alunos sem deficiência, mesmo que esta diferença não seja significante, devemos refletir sobre as condições e oportunidades que os alunos com deficiência física encontram no ambiente escolar para realizar as atividades propostas.

No contexto de aprendizagem, os alunos que percebem a si mesmos como altamente competentes em uma atividade ou habilidade tendem a persistir por mais tempo na tarefa ou ação e continuar em tentativas de executar a habilidade com maestria, mesmo quando encontram dificuldades. Ao contrário, os alunos que percebem a si próprios como pouco competentes tendem a desistir ou perdem o interesse no desenvolvimento de habilidades ou tarefas, principalmente quando encontram dificuldades ${ }^{23}$.

O conhecimento a respeito da autopercepção de competência que os alunos com deficiência possuem pode auxiliar os professores a subsidiar intervenções com atividades engajadas nas propostas de educação inclusiva, de forma a promover experiências variadas para a aquisição de habilidades, isto porque, à medida que alguém aumenta ou atinge metas pessoais ou demonstra aprimoramento individual, manifesta a sua competência, e, esta competência, quando percebida, encoraja a pessoa às novas experiências e ao aprimoramento daquilo que já foi aprendido ${ }^{24}$.

Ao compararmos a percepção de competência que os professores pesquisados possuem a respeito do grupo de alunos com deficiência e a autopercepção dos próprios alunos, o resultado obtido com o teste MannWhitney ( $\mathrm{p}=0.0503$ ) demonstrou que não houve diferença estatisticamente significante. No entanto, vale ressaltar que este valor está muito próximo da diferença estatisticamente significante e sendo assim, a avaliação da percepção do professor se diferenciou negativamente da avaliação 
de autopercepção dos alunos com deficiência. A fim de aprofundarmos a análise, buscamos por meio das medidas de dispersão obtidas no cálculo dos quartis estes valores e verificamos que todos os correspondentes à avaliação do professor são inferiores aos valores correspondentes à avaliação dos alunos. Dessa forma, podemos concluir que apesar da diferença entre as avaliações não ser considerada estatisticamente significante de acordo com o teste MannWhitney, as percepções de competência dos professores com relação aos alunos com deficiência são desfavoráveis quando comparada à autopercepção de competência do mesmo grupo de alunos.

$\mathrm{Na}$ análise comparativa da percepção de competência que os professores pesquisados possuem a respeito do grupo de alunos sem deficiência e a autopercepção de competência dos mesmos alunos, foi constatado que não houve a diferença estatisticamente significante $(\mathrm{p}=0.8633)$. Sendo assim, buscamos a análise por meio das medidas de dispersão obtidas no cálculo dos quartis. Verificamos que os valores correspondentes à avaliação do professor são iguais aos valores correspondentes à avaliação dos alunos, com exceção do valor da mediana que na avaliação do professor é superior à mediana obtida na avaliação dos alunos. Dessa forma, podemos concluir que a análise dos quartis confirma o resultado de diferença estatisticamente não significante apontada no teste Mann-Whitney e que a única variação presente na comparação dos quartis demonstra uma avaliação favorável do professor comparada à avaliação do grupo de alunos sem deficiência. Mais uma vez recorrendo aos valores de escores brutos obtidos no PEGS dos professores e dos alunos, observamos que as avaliações dos professores e dos alunos sem deficiência mantém um padrão mais homogêneo de valores relacionados às suas percepções de competência. No entanto, identificamos que os alunos com deficiência, tanto na perspectiva dos alunos quanto de seus professores, apresentaram um resultado mais heterogêneo refletindo as características individuais encontradas neste grupo.

A outra questão que nos chama a atenção é o fato de haver proximidade dos valores apresentados na análise das medidas de dispersão entre a percepção do professor e a autopercepção dos alunos sem deficiência, diferente do que ocorreu entre a percepção dos professores e a autopercepção dos alunos com deficiência. Uma hipótese a ser destacada é a possibilidade da percepção do professor com relação à competência dos alunos influenciar a autopercepção do grupo sem deficiência por meio das suas atitudes sociais e feedback oferecido diante do desempenho dos alunos sem deficiência ao realizarem as atividades escolares. Já com relação ao grupo de alunos com deficiência essa hipótese não se sustenta, na medida em que a autopercepção dos alunos é mais favorável do que a percepção que os professores possuem a respeito da competência desses alunos para realizar as atividades escolares. Outras hipóteses emergem a partir dessas considerações. Os alunos do grupo com deficiência teriam uma autopercepção incompatível com sua competência real ao realizar as atividades no contexto escolar? Os professores por meio de suas atitudes sociais frente ao grupo de alunos com deficiência estariam oferecendo feedback a respeito da competência desses alunos no sentido de buscar seu desenvolvimento e superar as dificuldades propostas? Os professores teriam sua percepção de competência desse grupo de alunos influenciada pelo estigma da deficiência, e, com isto, a sua percepção estaria incompatível com a competência real desses alunos ao realizarem as atividades escolares? Os alunos com deficiência física estão tendo as mesmas oportunidades de participação e realização de atividades no ambiente escolar a ponto da sua competência ser observada e analisada por seus professores, ou estariam sendo privados de tais experiências a partir do julgamento prévio de incapacidade pelo meio social no qual estão inseridos? Diante da natureza e objetivos deste estudo, estas questões não poderão ser respondidas neste momento, no entanto, podem ser consideradas como perguntas disparadoras da inquietação própria do pesquisador, para que novos estudos possam ser desenvolvidos, a fim de contribuir para a pesquisa na área da inclusão escolar.

\section{CONCLUSÃO}

A partir dos resultados obtidos neste estudo foi constatado que a presença da deficiência física influiu desfavoravelmente na percepção de competência tanto na perspectiva dos professores quanto na dos próprios alunos com essa condição. Sendo assim, diante da presença de disfunções motoras, fica evidente a necessidade de possibilitar modificações nas tarefas e no ambiente para favorecer a funcionalidade do aluno ao realizar as atividades escolares propostas. Uma vez que existe uma demanda do ambiente incompatível com as habilidades do aluno ao mesmo tempo em que as modificações e adequações não são valorizadas no ambiente, inevitavelmente haverá prejuízo no desempenho e participação desses alunos.

O estudo aponta para hipóteses a serem testadas, considerando a possibilidade de influência da percepção do professor sobre a trajetória escolar do aluno com deficiência, e sobretudo, levando em conta o sentido da avaliação de competência, sob parâmetros do próprio indivíduo e padrões sociais, na construção de histórias de vida. 
Andrade MMA, et al. Percepção de competência, em situação de atividades. Rev Ter Ocup Univ São Paulo. 2018 set.-dez.;29(3):246-53.

PARTICIPAÇÃO DAS AUTORAS: Mirela Moreno de Almeida Andrade - Discente responsável pela pesquisa. Camila Boarini dos Santos - Discente auxiliar da pesquisa. Rita de Cássia Tibério Araújo - Orientadora.

\section{REFERENCIAS}

1. Lopez BP, Molina PD, Arnaiz BN. Competencia ocupacional y disfunción ocupacional. In: Molina PD, Arnaiz BN. Conceptos fundamentales de terapia ocupacional. Madrid: Medica Panamericana; 2001. p.25-34.

2. White RW. Motivation reconsidered: the concept of competence. Psychol Rev. 1959;30:297-333. http://dx.doi. org/10.1037/h0040934.

3. Trombly CA, Radomski MV. Terapia ocupacional para disfunções físicas. 5a ed. São Paulo: Santos; 2005.

4. Phillips DA, Zimmerman M. The development course of perceived competence and incompetence among children. In: Sternberg R, Kolligian J, editors. Competence considered. New Haven: Yale University; 1990.

5. Phillips DA. The illusion of incompetence among academically competent children. Child Dev. 1994;55:2000-16.

6. Bandura A. Self-efficacy: the exercise of control. New York: Freeman and Company; 1997.

7. Faria MGA. Concepções pessoais de competência: promover a aprendizagem e o desempenho dos alunos. Inovação (Maranhão). 1998;11(2):47-55.

8. Langer EJ. The Illusion of incompetence. In: Perlmutter LC, Monty RA, editors. Choice and perceived control. New York: Hillsdale; 1979.

9. Langer EJ, Park K. Incompetence: a conceptual reconsideration. In: Sternberg R, Kolligian J, editors. Competence considered. New Haven: Yale University; 1990.

10. Ferreira EEB. A Percepção de competência, autonomia e pertencimento como indicadores da qualidade motivacional do aluno [tese]. Marília: Faculdade de Filosofia e Ciências, Universidade Estadual Paulista, UNESP; 2010.

11. Omote S, Oliveira AAS, Baleotti LR, Martins SESO. Mudanças de atitudes sociais em relação à inclusão. Paidéia Cad Psicol Educ (Ribeirão Preto). 2005;15(32):387-98. http:// dx.doi.org/10.1590/S0103-863X2005000300008.

12. Alahbabi AK. K-12 special and general education teachers' attitudes toward the inclusion of students which special needs in general educational classes in The United Arab Emirates (UAE). Int J Spec Educ. 2009;24(2):42-54.

13. Brito MC. Síndrome de Asperger e educação inclusiva: análise de atitudes sociais e interações sociais [tese]. Marília: Faculdade de Filosofia e Ciências, Universidade Estadual Paulista, Marilia; 2011. Disponível em: http://hdl.handle. net/11449/102190.

Submetido em: 14.10 .18

Aceito em: 03.01.19
14. Janes CRXF, Omote S. Atitudes sociais em relação à inclusão: o curso de pedagogia da faculdade de ciências e tecnologia da unesp. Nuances Estud Educ (Presidente Prudente). 2013;24(2):158-73. http://dx.doi.org/10.14572/nuances. v24i2.2486.

15. Jobe D, Rust JO, Brissie J. Teacher attitudes toward inclusion of students with disabilities into regular classrooms. Education (Barcelona). 1996;117(1):148-54.

16. Hastings RP, Oakford S. Student teachers attitudes toward the inclusion of children with special needs. Educ Psychol (Washington). 2003;23(1):87-94. https://doi. org/10.1080/01443410303223.

17. Triviños ANS. Introdução à pesquisa em ciências sociais: a pesquisa qualitativa em educação. São Paulo: Atlas; 1987.

18. Missiuna C, Pollock N, Law M. Perceived efficacy and goal setting system (PEGS). San Antonio, TX: Psichological Corporation; 2004.

19. Missiuna C, Pollock N, Law M, Walter S, Cavey N. Examination of the perceived efficacy and goal setting system (PEGS) with children with disabilities, their parents, and teachers. Am J Occup Ther. 2006;60:204-14. doi: 10.5014/ ajot.60.2.204.

20. Ruggio CIB. Adaptação transcultural do perceived efficacy and goal setting system - PEGS para crianças brasileiras [dissertação]. Belo Horizonte: Universidade Federal de Minas Gerais; 2008. Disponível em: http://hdl.handle.net/1843/ MSMR-7WFPC6.

21. Pelosi MB, Nunes LRDP. A ação conjunta dos profissionais da saúde e da educação na escola inclusiva. Rev Ter Ocup Univ São Paulo. 2011;22(1):52-9. https://doi.org/10.11606/ issn.2238-6149.v22i1p52-59.

22. Almeida G, Valentini N, Berleze A. Um estudo com crianças e adolescentes do Ensino Fundamental. Rev Movimento Ver Educ Fisica UFRGS. 2009;15(1):71-97. doi: https://doi. org/10.22456/1982-8918.2416.

23. Valentini NCA. Influencia de uma intervenção motora no desempenho motor e na percepção de competência de crianças com atrasos motores. Rev Paul Educ Fisica (São Paulo). 2002;16(1):61-75. doi: https://doi.org/10.11606/issn.25945904.rpef.2002.138698.

24. Gallahue D, Donnelly FC. Educação física desenvolvimentista para todas as crianças. 4a ed. São Paulo: Phorte; 2008. 